\title{
Tendências recentes da investigação internacional sobre pedagogia do ensino superior: uma revisão da literatura
}

\author{
Jorge Ramos do Ó, Marta Almeida, \\ Joana Viana, Tatiana Sanches \& Ana Paz
}

Resumo:

O artigo visa analisar a expressão que a pedagogia do ensino superior vem assumindo na investigação. Para esse efeito, tomou-se como ponto de partida e inspiração metodológica o trabalho realizado por Kuzhabekova, Hendel \& Chapman (2015). Neste, os autores analisam a produção científica entre 2002 e 2011, tendo como foco as publicações que se dedicam a temáticas sobre o ensino superior. No estudo são elencadas as revistas com maior número de artigos neste âmbito; os anos com mais publicações, os investigadores mais prolixos, apresentando ainda um mapeamento dos países de origem e afiliação institucional. No trabaIho ora apresentado, afunilou-se a temática, circunscrevendo a análise aos textos que versam sobre pedagogia do ensino superior, área a requerer um investimento acentuado, quer pelos desafios que se colocam atualmente a este nível de ensino, quer pela necessidade de (re)pensar a formação pedagógica dos docentes que aí atuam. Para este efeito, a análise compreendeu uma pesquisa exaustiva dos artigos publicados entre 2012 e 2018, nas dez principais publicações identificadas no estudo anterior. Os resultados mostram que o pico de publicações se deu em 2014, tendo vindo a decrescer nos últimos anos, sendo a revista que mais se destaca a Teaching in Higher Education. A maior percentagem de artigos provém do Reino Unido, país com grande tradição na formação pedagógica dos seus docentes, seguindo-se a Austrália e os EUA.

Palavras-chave:

pedagogia do ensino superior; ensino superior; investigação. 


\title{
Recent trends of international research on higher education pedagogy: a literature review
}

\begin{abstract}
The article aims to analyze the expression that higher education pedagogy has been assuming in research. For this purpose, the work done by Kuzhabekova, Hendel \& Chapman (2015) was taken as a starting point and methodological inspiration. In this paper, the authors analyze the scientific production between 2002 and 2011, selecting publications that focus on the themes of higher education. In the study, the journals with the highest number of articles in this field are listed, the years with the most publications, the most prolix researchers, and a mapping of their countries of origin and institutional affiliation. In the work presented here, the topic was narrowed down, circumscribing the analysis to texts that deal with pedagogy of higher education, an area that requires a significant investment, both by the challenges that are currently posed at this level of education, and by the need to (re)think about the pedagogical formation of the teachers who work there. For this purpose, the analysis comprised an exhaustive survey of the articles published between 2012 and 2018, in the ten main publications identified in the previous study. The results show that the peak of publications occurred in 2014, having declined in recent years, with the most prominent magazine being Teaching in Higher Education. The highest percentage of articles comes from the United Kingdom, a country with a long tradition in the pedagogical training of its teachers, followed by Australia and the USA.
\end{abstract}

Keywords: higher education pedagogy; higher education; research.

\section{Tendances récentes de la recherche internationale sur la pédagogie de l'enseignement supérieur: une revue de la littérature}

Resumé: Cet article propose analyser l'expression que la pédagogie de l'enseignement supérieur a donnée dans la recherche. Pour ce but, le travail réalisé par Kuzhabekova, Hendel \& Chapman (2015) a été pris comme point de départ et inspiration méthodologique. Dans ce travail, les auteurs analysent la production scientifique entre 2002 et 2011, en se concentrant sur les publications consacrées à la thematique de l'enseignement supérieur. L'étude présente les revues qui contiennent le plus grand nombre d'articles dans ce domaine; les années avec plus de publications, les chercheurs les plus prolixes, ainsi qu'une cartographie des pays d'origine et de l'affiliation institutionnelle. Dans ce travail, le thème s'est rétréci en se limitant aux textes traitant de la pédagogie de l'enseignement supérieur, domaine à investir fortement, tant par les défis qui se posent actuellement à ce niveau d'enseignement, soit par la nécessité de (ré)penser la formation pédagogique des enseignants qui y travaillent. Pour cela, l'analyse comprenait une recherche approfondie des articles publiés entre 2012 et 2018 dans les dix principales publications identifiées dans l'étude précédente. Les résultats montrent que le pic de publications a été atteint en 2014 et qu'elles ont diminué ces dernières années, le magazine le plus remarquable étant Teaching in Higher Education. La plus grande partie des articles provient du Royaume-Uni, pays qui a une large tradition sur la formation pédagogique de ses enseignants, suivie de l'Australie et des États-Unis.

Mots clés: pédagogie de lienseignement supérieur; enseignement supérieur; recherche.

\section{Tendencias recientes de la investigación internacional sobre la pedagogía de la enseñanza superior: una revisión de la literatura}

Resumen: El artículo busca analizar la expresión que la pedagogía de la enseñanza superior viene asumiendo en la investigación. Para ello, se tomó como punto de partida e inspiración metodológica el trabajo realizado por Kuzhabekova, Hendel \& Chapman (2015). En este trabajo, los autores analizan la producción científica entre 2002 y 2011, teniendo como foco las publicaciones que se dedican a temáticas sobre la enseñanza superior. En el estudio, se enumeran las revistas con mayor número de artículos en este ámbito, los años con más publicaciones, los investigadores más prolíficos, presentando un mapeamiento de sus países de origen y afiliación institucional. En el trabajo presentado, se afinó la temática, circunscribiendo el análisis a los textos que versan sobre pedagogía de la enseñanza superior, área a requerir una inversión acentuada, tanto por los desafíos que se plantean actualmente a este nivel de enseñanza, o por la necesidad de (re)pensar la formación pedagógica de los docentes que allí actúan. Para ello, el análisis comprendió una investigación exhaustiva de los artículos publicados entre 2012 y 2018 , en las diez principales publicaciones identificadas en el estudio anterior. Los resultados muestran que el pico de publicaciones se dio en 2014, habiendo decrecido en los últimos años, siendo la revista que más se destaca la Teaching in Higher Education. El mayor porcentaje de artículos proviene del Reino Unido, país con gran tradición en la formación pedagógica de sus docentes, seguido de Australia y Estados Unidos.

Palabras clave: pedagogía de la enseñanza superior; enseñanza superior; investigación. 


\section{Introdução}

O artigo visa analisar a expressão que a pedagogia do ensino superior revela na investigação a nível internacional, procurando perceber qual o lugar que este domínio vem conquistando nos últimos anos, cientes de que esta área requer um investimento acentuado, quer pelos desafios que se colocam atualmente a este nível de ensino, quer pela necessidade de (re)pensar a formação pedagógica dos docentes que aí atuam. Com efeito, no momento em que assumem o seu posto na universidade, os novos professores já têm atrás de si uma carreira, se assim se pode dizer, de vários anos como investigadores. Se é certo que essa trajetória apresenta exigências de performance elevadas, não é menos verdade que a proficiência na investigação não se adapta de per se às tarefas de ensino que têm de passar a desempenhar e que Ihes demandam, além disso, múltiplas competências que não tiveram oportunidade de desenvolver previamente. A relação instável, e não raro tensa, entre ciência e ensino é, sem dúvida, uma das marcas mais visivelmente persistentes na história da universidade desde o início do século XIX.

Acresce que os professores universitários têm hoje pela frente significativos desafios, que resultam em larga medida: (i) do afluxo em grande número de estudantes à graduação e pós-graduação, provenientes de trajetórias escolares muito diversificadas, com expectativas e taxas de sucesso assaz dissemelhantes; (ii) do trânsito e da própria velocidade com que os saberes se cruzam e se consolidam para lá da taxonomia e das fronteiras disciplinares clássicas; (iii) da crescente demanda pela integração de novas tecnologias e da circulação dos alunos em vários ambientes de aprendizagem. Este contexto exige a cada professor que se oriente para vários objetivos e não apenas para um, assim como a sua prática deve atender a múltiplos racionais. Dir-se-á então que hibridização, variabilidade, flexibilidade e autorreflexão serão hoje, em síntese, os rótulos que melhor expressam as competências requeridas globalmente aos professores de ensino superior (ES) na atualidade.

Neste cenário, importa perceber que atenção vem sendo dada pela comunidade científica à área da pedagogia do ensino superior, produzindo conhecimento que robusteça a sua afirmação enquanto campo de saber, e que sustente uma intervenção ao nível da formação e desenvolvimento profissional dos académicos.

No trabalho ora apresentado assume-se como referência e inspiração metodológica a pesquisa realizada por Kuzhabekova, Hendel \& Chapman (2015). Nela os autores analisam a produção científica entre 2002 e 2011, listando e hierarquizando as publicações que se dedicam a temáticas sobre ES. Nesse estudo são elencadas as revistas com maior número de artigos neste âmbito, os anos com mais publicações e os investigadores mais prolixos, apresentando-se ainda um mapeamento dos países de origem dos autores e respetiva afiliação institucional. 
Face ao exposto, e na esteira do trabalho supracitado, optou-se por afunilar a temática em análise, circunscrevendo o foco aos textos que versam sobre pedagogia do ensino superior. Procedeu-se a uma pesquisa exaustiva dos artigos publicados entre 2012 e 2018 nas dez principais publicações identificadas por Kuzhabekova et al. (2015), em função da atenção dedicada à temática e com os objetivos de perceber como esse interesse vai variando ao longo dos anos, identificar os autores com mais investigação publicada na área, e mapear a sua afiliação institucional e país de origem. Apresenta-se, para além da introdução, o enquadramento do estudo, a metodologia, os resultados e sua discussão, terminando-se com algumas considerações finais.

\section{Enquadramento}

\section{A relevância da formação pedagógica dos docentes na Universidade de hoje}

$\mathrm{Na}$ literatura a necessidade de formação pedagógica dos professores do ensino superior parece indubitável (e.g., Cruz Tomé, 2003; Cunha, 2010; Davidson, 2004; De Ketele, 2003; Elton, 2009; Esteves, 2010; Leite \& Ramos, 2010; Tardif, 2000; Zabalza, 2004, 2007), no entendimento de que "a docência universitária reclama requisitos que vão para além dos relativos aos campos disciplinares a que cada docente se encontra vinculado" (Leite \& Ramos, 2010, p. 35). Zabalza (2004, 2007), por exemplo, afirma que o exercício profissional docente corresponde a uma atividade profissional complexa, reclamando uma formação específica pois "esse é o nosso trabalho e levá-lo a cabo em boas condições exige saber sobre ele, sobre a sua teoria e a sua prática com uma certa profundidade" (Zabalza, 2007, p. 70).

Também nos movimentos gerados no interior dos estabelecimentos de ensino superior se sustenta a ideia de que a formação pedagógica dos docentes deve ser uma preocupação institucional. São disso reflexo os programas de formação de professores universitários que fazem parte da paisagem habitual do ES nos países de língua inglesa desde o termo da década de 1960. Estudos como o de Gibbs e Coffey (2004), que analisa os efeitos da formação pedagógica dos docentes em 22 instituições de ES de oito países, reforçam a ideia de que esta se reflete positivamente nas práticas dos professores e nas aprendizagens dos estudantes.

Cabe reconhecer que a crescente atração pela melhoria do ensino e da aprendizagem no ES se sustenta numa multiplicidade de disciplinas e provém também de soluções organizacionais diferenciadas. Torna-se pois necessário mergulhar na história e atender aos figurinos e respetivas variações internacionais, a fim de fornecer um contexto que efetivamente possa dar conta da importante vitalidade do campo ensino e aprendizagem universitária em todo o mundo.

Para compreender a relevante questão das políticas públicas e do financiamento que acompanha a formação de professores universitários, recorremos à informação 
produzida pelo ICED - International Consortium for Educational Development, uma prestigiada organização mundial de ES. Em 2013, por ocasião da passagem dos 20 anos da sua fundação, produziu um relatório que se intitula The preparation of University teachers internationally report (ICED, 2014), identificando as políticas e práticas em atividade. Percebe-se aí que um espetro muito largo de países tem desenvolvido ou começa a implementar políticas para melhorar os standards de performance dos docentes do ES. O relatório apresenta os resultados de uma pesquisa sistemática num conjunto alargado de países, a saber: África do Sul, Alemanha, Austrália, Bélgica, Canadá, Croácia, Dinamarca, Espanha, Estados Unidos da América, Estónia, Etiópia, Finlândia, Holanda, Irlanda, Israel, Índia, Japão, Noruega, Reino Unido, Sri Lanka, Suécia, Suíça, Tailândia. Em alguns países, a política nacional ou regional é um requerimento legal. Um exemplo é o Sri Lanka, onde se implementou em 1997 uma política que demanda que os professores recém-recrutados obtenham uma qualificação credenciada pela instância nacional University Grants Commission. A Noruega mantém, também desde há longa data, uma regulamentação nacional que impõe a qualificação dos professores universitários para a docência. A Etiópia segue uma política nacional desde 1994, exigindo aos professores de todos os níveis, do básico ao superior, a posse da qualificação de formação necessária para garantir o emprego docente. Na Dinamarca, depois de 1993, com a publicação de um memorandum ministerial sobre a carreira académica, todos os assistentes universitários estão obrigados a seguir um programa de formação. Na Finlândia, o envolvimento em estudos pedagógicos é requerido nos institutos politécnicos. Na Suíça, o sistema nacional requer uma formação docente dos professores universitários nas Universidades de Ciências Aplicadas. Nos restantes casos contemplados pelo relatório ICED (2014), as políticas nacionais ou regionais não são mandatórias. No Reino Unido um quadro nacional para normas profissionais foi introduzido, mas não é juridicamente vinculativo, sendo antes "propriedade" do sector do ES, existindo um "mandated development of teaching and learning units or provided incentive funding for the development of such centers" (Lewis, 2010, p. 15). Em 1997, o relatório do National Committee of Inquiry into Higher Education, também conhecido por Dearing Report (Dearing, 1997), constituiria um marco da maior relevância e que afetaria o futuro do faculty development no Reino Unido.

Em 2008, as universidades de investigação da Holanda assinaram o Mutual Agreement of University Teaching Qualification (VSNU, 2008), garantindo que cada universidade se adequa aos normativos e exigências da University Teaching Quality (UTQ), embora sem assegurar a qualidade da sua aplicação. A UTQ holandêsa é obrigatória, não através de determinação estatal, mas antes acordada por autorregulação e assinada por todos os vice-chanceleres. Cada universidade descreve os seus próprios standards para a UTQ, conquanto esteja disseminado o uso de um perfil contendo cinco competências. A associação holandesa das universidades de ciências aplicadas 
(Vereniging van Hogescholen) concordou, em 2006, em implementar também um sistema de qualificação do ensino. Já em 2018, no mesmo país, é feito um balanço dos dez anos da aplicação daquele standard, também conhecido como BKO (VSNU, 2018). Na Suécia, e entre 2005 e 2010, foi introduzida na legislação a formação obrigatória dos docentes de ES, mas as universidades gozam de autonomia para a concretizar, tendo a associação de reitores aprovado diretrizes propostas pela SWEDNET, que se tornaram no standard nacional. No Japão, em 2007, uma associação de universidades pugnou pelo estabelecimento de standards pedagógicos para o ensino de $1^{\circ}$ ciclo - exigindo do governo a criação de escolas de pós-graduação para a formação de novos professores -, o que não se viria a realizar, de modo que, em muitas das universidades do país, a decisão de receber este tipo de formação é facultativa. No entanto, data dessa altura uma portaria ministerial do MEXT - a sigla do Ministério da Educação, Cultura, Desporto, Ciência e Tecnologia - que especifica a norma pedagógica mínima exigida para se ensinar na universidade. Na Tailândia a formação de professores do ES não é obrigatória, mas aplicam-se normas nacionais para cursos com essa finalidade. Não existe uma obrigatoriedade nacional para a formação de professores do ES nos EUA, exceto que o corpo docente de pós-graduação deve receber um mínimo de um dia de formação, credenciada por organismos regionais. Em 2014, o National Forum for Teaching and Learning, da Irlanda, começou a desenvolver um quadro nacional para o desenvolvimento profissional de professores do ES. Desde 2011, na Austrália existe o Higher Education Standards Framework que é, desde então, aplicado aos certificados de pós-graduação em ensino e aprendizagem, de acordo com a política nacional que regula a educação e formação do país. Na Alemanha, existe também um quadro normativo para as suas quase 400 universidades, determinando que a capacidade pedagógica deve ser avaliada no processo de admissão de novos professores. Cada estado federal interpreta a lei-quadro, o que resulta numa implementação diferenciada.

Atente-se, agora, na cronologia da formação em pedagogia do ensino superior. Em vários países, a constituição de um ou mais centros de desenvolvimento, programas ou comités far-nos-á recuar até aos alvores da década de 1960. O primeiro faculty development center nos Estados Unidos foi o Center for Research on Learning and Teaching, criado em 1962 na University of Michigan-Ann Arbor (Ouellett, 2010). Embora existissem menos de cinquenta programas de desenvolvimento no final da década (Sullivan, 1983), por volta de 1975, 60\% de todas as instituições de ES norte-americanas indicaram que tinham organizado os seus faculty development programs (Centra, 1976). Em concreto, uma pesquisa de 1986 descobriu que 44\% de todas as four-year institutions (including large and small, private and public) tinham programas de desenvolvimento de professores, enquanto, uma década mais tarde, Crawley descobriu que 64\% das 104 universidades de pesquisa (de acordo com a classificação da Carnegie Foundation for the Advancement of Teaching) tinha um coordenador ou diretor de um 
"faculty or instructional development program or unit" (Lewis, 2010, p. 17). Hoje, nos Estados Unidos, assiste-se a um crescendo nos faculty development programs e a um aumento do número de indivíduos que identificam a pedagogia do ES como o seu campo investigação, evidência esta que é demonstrada pelo trabalho de Sorcinelli et al. (2006), e pelo Professional and Organizational Development Network's New Developers Pre-Conference Workshop de 2008 e 2009. No Reino Unido, a primeira unidade de desenvolvimento educacional em ES foi fundada em Surrey in 1967. Na viragem para os anos 1990 existiam noventa outras suas congéneres, e, pelo final de 2005, setenta e cinco. A razão desta aparente quebra é que instituições de mais pequena dimensão criaram um comité de ensino e aprendizagem - em vez de uma unidade de desenvolvimento educacional organizada -, através do qual passaram a ser coordenadas as atividades e os programas educativos.

"The call for research into teaching methods and into causes for wastage and high failure rates made by committees" levaram algumas universidades, na Austrália e também por meados dos anos 1960, "to establish, by various names, academic or educational development units with their primary function the improvement of teaching", refere Moses (1987, p. 454) num dos primeiros estudos comparados sobre esta área. Em alguns casos, as solicitações realizadas por alunos levaram à criação de academic development units numa série de instituições. No início da presente década, existiam vinte e cinco instituições na Austrália, seis na Nova Zelândia, e uma nas Fiji, com "academic development units" (Lewis, 2010, pp. 17-18).

\section{A construção do conhecimento em torno da pedagogia do ensino superior}

$\mathrm{O}$ artigo Mapping global research in international higher education, publicado em 2015 por Alia Kuzhabekova, Darwin Hendel e David Chapman, fornece-nos uma visão panorâmica do campo. Trata-se de um trabalho que deita mão da bibliometria e da análise de conteúdo como metodologias cruzadas para identificar investigadores, instituições, países e áreas científicas que contribuem para a pesquisa em ES, ao mesmo tempo que estabelece padrões de conetividade entre autores, revistas, disciplinas e tópicos.

A base de dados que estes autores construíram identifica um conjunto impressivo de mais de 2300 publicações da Web of Science, no período entre 2002 e 2011, relativas à investigação em ES. O estudo mostra que o número de trabalhos cresceu exponencialmente de um total de 68 em 2002 para 472 em 2012, verificando, ao mesmo tempo, que as redes entre investigadores continuam a operar no interior das fronteiras nacionais e que são ainda dominadas por um pequeno número de países ocidentais: enquanto os 3362 autores identificados provinham de 1164 diferentes instituições dispersas pelo mundo, só cerca de $11 \%$ dos artigos era subscrito por investigadores de mais que uma nacionalidade (Kuzhabekova et al., 2015). Não obstante esta evidência 
da fragilidade das redes de cooperação internacional, é facto que o corpo de literatura atinente ao tópico dos estudos comparados em ES tem conhecido um rápido crescimento no passado recente. E, para este particular, serve-nos a informação carreada por Altbach (2009), fundador e diretor do Center for International Higher Education na Lynch School of Education at Boston College, que, apenas em referência ao ano de 2008 e às mais proeminentes publicações periódicas, registou 228 artigos provenientes de 30 diferentes países. O mesmo quadro de registos construído por Kuzhabekova et al. (2015) revelava que 92 países estão representados, isto é, quase metade da totalidade dos que existem no mundo. Mostrava ainda que é no Reino Unido que mais se produzem estudos sobre ES - cerca de um quarto do total -, seguido dos Estados Unidos (14,9\%), da Austrália (13,9\%), da África do Sul e da China que contribuem ambos com cerca de 6\%. Os restantes países da Europa Ocidental e o Canadá não vão além dos de $4 \%$ no conjunto da investigação.

Os investigadores identificados na base de dados provêm de 1164 diferentes instituições. A tendência geral mantém-se com a prevalência das universidades do Reino Unido, da Austrália e da África do Sul. Talvez a surpresa maior seja a de que apenas duas instituições norte-americanas surgem no top 15, enquanto a Open University que trabalha predominantemente com ensino à distância, tecnologia e educação - se impõe como a terceira. A quinta posição que a Universidade de Hong Kong ocupa expressa, evidentemente, o interesse que a China está a dar ao ES.

Olhamos em seguida para os investigadores com maior produção no campo do ES que Kuzhabekova et al. (2015) identificaram, a partir do conjunto vasto de 3362 registos de autores com mais de cinco publicações. Como se verifica, os nomes mais presentes no campo vêm de uma panóplia de nações muito mais ampla do que aquela que as universidades refletem, como é o caso de Tomusk que se encontra a trabalhar na Hungria, país sem tradição na pesquisa em ES. Os autores mais citados no campo e nas organizações do ES são, como seria de esperar, aqueles que têm também um maior número de publicações: Philip Atlbach, do Boston College Center for International Higher Education, Simon Marginson (Universty College de Londres) e Burton Clark (UCLA).

Se atendermos agora às publicações periódicas que se dedicam à divulgação da investigação internacional em ES, fica bastante clara a pujança do campo ante a variedade de títulos. Kuzhabekova et al. (2015) identificaram 42 journals com grande consistência e influência num conjunto total de 318 registos. A Higher Education e a Studies in Higher Education, com origem na Society for Research into Higher Education (SRHE), lideram destacadamente e são as que permitem melhor e mais rapidamente compreender quer a geografia de temas e problemas, quer os novos movimentos e direções da investigação. Largamente, e sem surpresa, a palavra-chave que identifica a produção é teaching and learning, mas há um conjunto de outros tópicos com alguma 
consistência, e que parecem configurar as tendências do devir da pesquisa nesta ordem de importância: critical thinking, academic writing, collaborative learning, problem based learning, service learning (Kuzhabekova et al., 2015).

\section{Metodologia}

Tomando como referência e inspiração metodológica o trabalho realizado por Kuzhabekova et al. (2015), o presente estudo compreende a pesquisa exaustiva de todos os artigos publicados nas dez principais publicações (Journals) aí identificadas (Tabela 1), tendo agora como referência temporal os anos de 2012 a 2018 e focalizando-se apenas na temática pedagogia do ES.

Tabela 1. Ranking das publicações (Kuzhabekova et al., 2015)

\begin{tabular}{|c|c|}
\hline & REVISTA \\
\hline 1 & Higher Education \\
\hline 2 & Studies In Higher Education \\
\hline 3 & Journal of Higher Education \\
\hline 4 & Higher Education Policy \\
\hline 5 & Higher Education Research And Development \\
\hline 6 & European Journal of Education \\
\hline 7 & Higher Education Quarterly \\
\hline 8 & Research in Higher Education \\
\hline 9 & Teaching in Higher Education \\
\hline 10 & Journal of Education Policy \\
\hline
\end{tabular}

A recolha realizada foi conduzida durante os meses de novembro e dezembro de 2018, nas bases de dados agregadas pelo motor de busca EDS (Ebsco Discovery System), que compreende, para a Educação, as bases ERIC, Education Research Complete, Education Source e B-on. A pesquisa foi desenvolvida usando como critérios o termo higher education associado a outras palavras-chave, de modo a fazer combinações que pudessem devolver registos relativos à temática pedagogia do ES. Os termos associados a higher education foram: pedagogy, teaching, learning, teachers, professional development, educational development, faculty development e academic development. A pesquisa efetuou-se por descritor/palavra-chave e palavra no título do artigo. Os dados de identificação e descrição de cada um dos textos organizaram-se numa base de dados contendo: identificação da revista científica, ano de publicação, título do artigo, termos chave de pesquisa, nome do(s) autor(es), respetiva afiliação 
institucional e país. Após o terminus da pesquisa contabilizaram-se 628 artigos. Posteriormente procedeu-se a uma análise geral dos títulos e, quando necessário, procedeu-se a uma leitura flutuante dos resumos, para supressão daqueles que não se relacionassem com questões da pedagogia do ES, eliminando trabalhos que versavam sobre temas afins como, por exemplo, política educativa ou gestão e administração, entre outros. Após filtragem dos resultados, remanesceram 387 artigos (61.6\%) obedecendo aos critérios de análise selecionados.

\section{Apresentação e Discussão dos Resultados}

A pesquisa realizada nas dez publicações científicas apuradas (Tabela 1) em torno da temática da pedagogia do ES resultou na identificação de 387 artigos cuja distribuição por revista se apresenta no Gráfico 1.

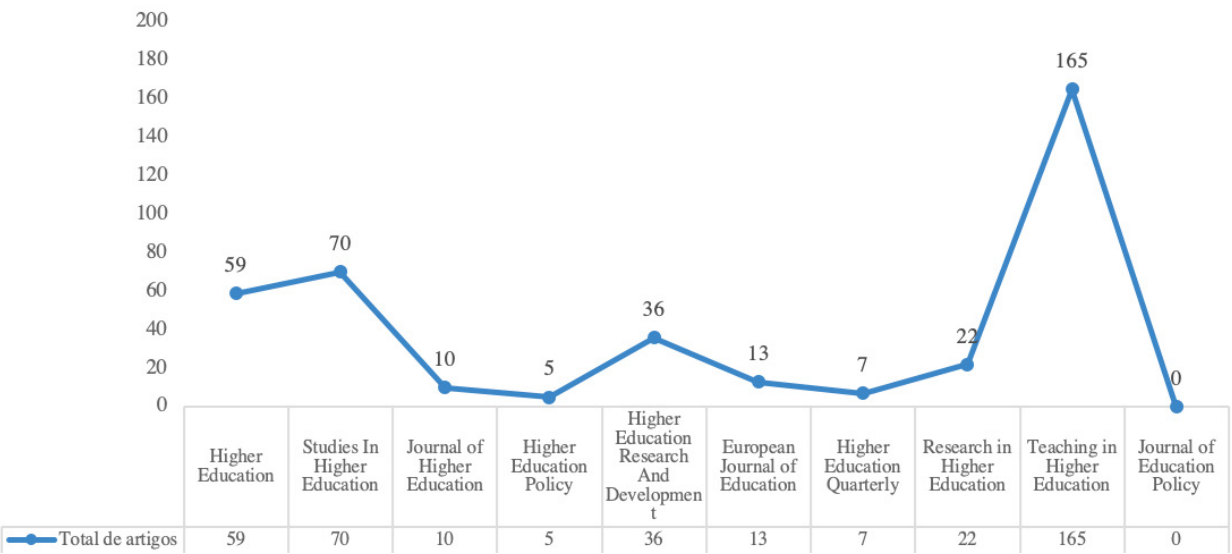

Gráfico 1. Número de artigos obtidos por publicação científica.

Como se pode apreciar, a revista que mais se destacou foi a Teaching in Higher Education, com 165 artigos (42\% do total de artigos publicados entre 2012 e 2018), o que seria expectável dada a própria designação que indicia um especial interesse pela temática da pedagogia do ES. Seguem-se as revistas Studies in Higher Education com 70 artigos (18\%) e Higher Education com 59 artigos (15\%). Apenas o periódico Journal of Education Policy não inclui qualquer artigo que se relacione com a temática em análise. 
Procurou-se, de seguida, identificar as palavras-chave mais associadas ao termo de pesquisa higher education, como ilustra o Gráfico 2.

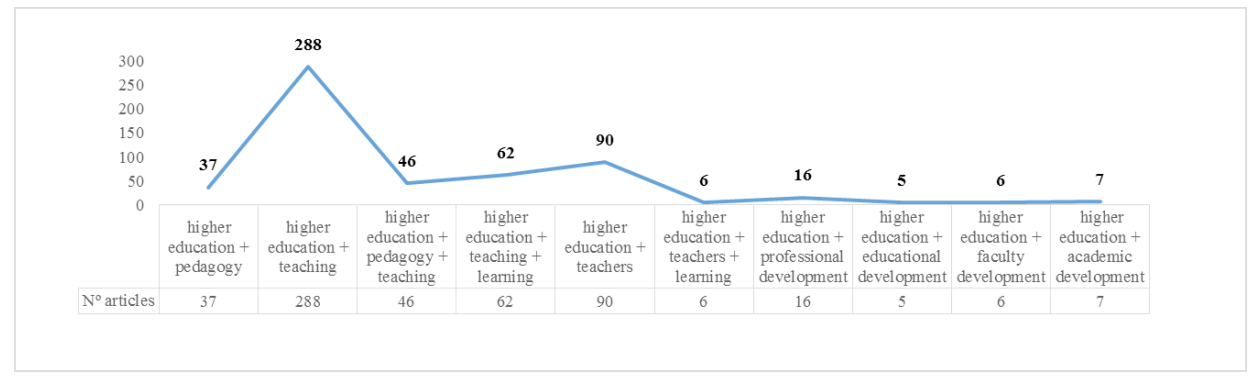

Gráfico 2. Número de artigos obtidos por termos de pesquisa.

A combinação que mais resultados devolveu foi higher education + teaching com 288 artigos (74\%), seguida de higher education + teachers com 90 artigos (23\%). Os termos combinados higher education + teaching + learning apresentam 62 resultados (16\%) enquanto o termo pedagogy com higher education devolve apenas 37 resultados $(9,6 \%)$. Termos tais como professional development, educational development, faculty development ou academic development devolvem-nos algumas entradas, ainda que residuais e, nessa perspetiva, foram também considerados. Na destacada publicação Teaching in Higher Education (Gráfico 1) são os termos higher education + teaching que apresentam mais resultados (116), como seria de esperar pelo próprio escopo da revista. No que se refere à combinação dos termos higher education + teachers surgem claramente menos resultados (15), até por comparação com outras revistas, como por exemplo a Higher Education e a Studies in Higher Education, o que se verifica na Tabela 2, que espelha a distribuição de artigos por revista em função dos termos de pesquisa. 
Tabela 2. Número de artigos obtidos por revista científica de acordo com os termos de pesquisa

\begin{tabular}{|c|c|c|c|c|c|c|c|c|c|c|}
\hline $\begin{array}{l}\text { Termos para Chave } \\
\text { de Pesquisa } \\
(2012-2018)\end{array}$ & 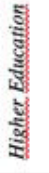 & 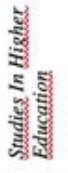 & 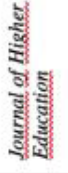 & 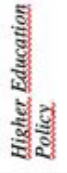 & 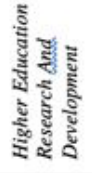 & 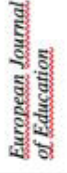 & 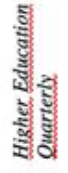 & 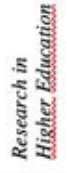 & 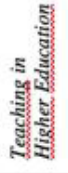 & 형 \\
\hline $\begin{array}{l}\text { "higher education" + } \\
\text { pedagogy }\end{array}$ & 2 & 3 & 0 & 0 & 0 & 1 & 0 & 0 & 31 & 0 \\
\hline $\begin{array}{l}\text { "higher education" + } \\
\text { teaching }\end{array}$ & 46 & 50 & 3 & 4 & 24 & 8 & 4 & 12 & 116 & 0 \\
\hline $\begin{array}{l}\text { "higher education" + } \\
\text { teaching + learning }\end{array}$ & 16 & 4 & 1 & 1 & 11 & 4 & 0 & 5 & 20 & 0 \\
\hline $\begin{array}{l}\text { "higher education" + } \\
\text { teachers }\end{array}$ & 24 & 19 & 6 & 1 & 3 & 3 & 3 & 16 & 15 & 0 \\
\hline $\begin{array}{l}\text { "higher education" + } \\
\text { "professional } \\
\text { development" }\end{array}$ & 4 & 1 & 0 & 0 & 7 & 0 & 0 & 0 & 4 & 0 \\
\hline $\begin{array}{l}\text { "higher education" } t \\
\text { "educational } \\
\text { development" }\end{array}$ & 0 & 0 & 0 & 0 & 4 & 1 & 0 & 0 & 0 & 0 \\
\hline $\begin{array}{l}\text { "higher education" + } \\
\text { "faculty } \\
\text { development" }\end{array}$ & 0 & 1 & 1 & 0 & 1 & 2 & 0 & 0 & 1 & 0 \\
\hline $\begin{array}{l}\text { "higher education" + } \\
\text { "academic } \\
\text { development" }\end{array}$ & 2 & 1 & 0 & 0 & 0 & 0 & 0 & 0 & 4 & 0 \\
\hline
\end{tabular}

Prosseguimos a análise da produção científica, procurando identificar a sua distribuição cronológica. É em 2014 (com 22,2\% dos artigos publicados) que se dá maior atenção a estas questões, decrescendo um pouco no ano seguinte $(17,8 \%)$ e posteriores (Gráfico 3). Com efeito, em 2017 e 2018 o número de artigos publicados fica abaixo dos registados em 2012, parecendo indiciar quer um esmorecimento do interesse dos investigadores pelas questões da pedagogia do ES, quer uma mudança nas políticas editoriais, que darão primazia a trabalhos sobre outras temáticas.

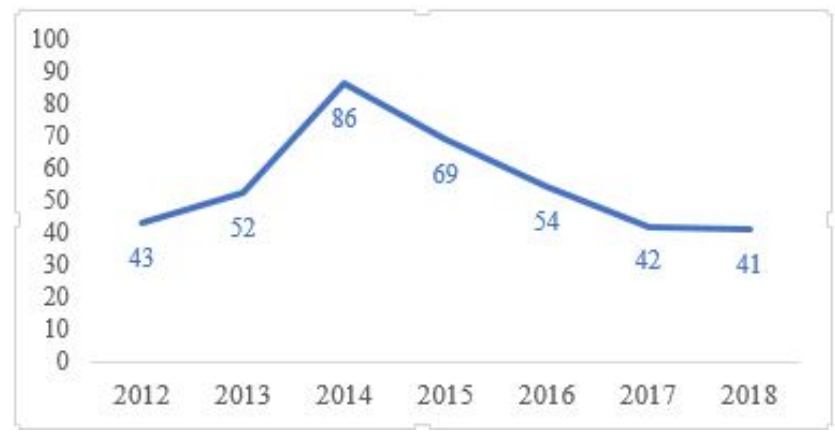

Gráfico 3. Número de artigos publicados entre 2012 e 2018. 
Analisando a origem dos 601 autores (considerando os $1^{\circ}, 2^{\circ}, 3^{\circ}, 4^{\circ}, 5^{\circ}, 6^{\circ}$ e $7^{\circ}$ autores) identificam-se 40 países. A maior percentagem de autores provém do Reino Unido (18,6\%), seguindo-se os de origem australiana (16,6\%) e americana (15,3\%) (Tabela 3). A representação de outros países é pouco expressiva, com percentagens inferiores a 7\%, o que coincide com a situação encontrada por Kuzhabekova et al. (2015), pese embora um ligeiro aumento de autores provenientes da Austrália face aos dos EUA. Dos restantes países, mantêm-se registos provenientes da África do Sul (6,5\%), da China (4,2\%) e também da Nova Zelândia (5.2\%), seguidos de outros países da Europa Ocidental e do Canadá (Tabela 3). Os artigos publicados cujos autores apresentam afiliação em Portugal situam o país na $13^{a}$ posição do ranking, com apenas $2,2 \%$ do total dos registos.

Tabela 3. Número de registos obtidos por país de afiliação dos autores

\begin{tabular}{|c|c|c|c|c|c|c|c|c|c|c|c|c|c|c|c|c|}
\hline o de & 过递 & 茎 & 出 & $\begin{array}{l}\frac{8}{8} \\
\text { 遂 }\end{array}$ & 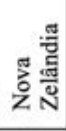 & 泀 & $\begin{array}{l}\text { 密 } \\
\text { 品 }\end{array}$ & 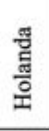 & 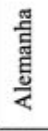 & 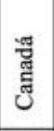 & 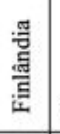 & $\begin{array}{l}\text { हू } \\
\text { हैं } \\
\text { Zे }\end{array}$ & 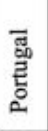 & 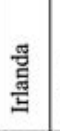 & 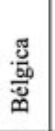 & 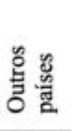 \\
\hline Total de registos & 112 & 100 & 92 & 39 & 31 & 25 & 22 & 21 & 20 & 19 & 15 & 13 & 13 & 11 & 8 & 60 \\
\hline$\%$ & 18,6 & 16,6 & 15,3 & 6,5 & 5,2 & 4,2 & 3,7 & 3,4 & 3,3 & 3,2 & 2,5 & 2,2 & 2,2 & 1,8 & 1,3 & 10 \\
\hline
\end{tabular}

*Apresenta-se apenas a lista dos 15 países com mais registos identificados entre os 40 países identificados nos 601 registos analisados, juntando-se os restantes paises da lista na categoria "outros paises".

Quanto à afiliação dos autores, identificam-se cerca de 600 instituições entre as quais prevalecem universidades da Austrália (48 autores), seguidas de universidades da África do Sul (26), do Reino Unido (25) e da Nova Zelândia (15). No cômputo geral, destacam-se a University of Sydney, Macquarie University, University of Western Cape, University of Otago, University of Queensland e University of Glasgow. Estes resultados coincidem com a situação encontrada por Kuzhabekova et al. (2015), apesar de se registar um aumento relevante de publicações cujos autores pertencem a universidades na África do Sul e na Nova Zelândia. Com valores próximos destas universidades surge também a University of Hong Kong, o que confirma as tendências identificadas anteriormente por Kuzhabekova et al. (2015) e o florescente interesse pelo tema na China.

Entre os 878 registos de autores, registam-se cinco investigadores com três ou mais publicações entre 2012 e 2018, a saber: Mary Ryan (Austrália), Angela Brew (Austrália), Ann Stes (Bélgica), Nico Verloop (Holanda), Gerda Visser-Wijnveen (Bélgica), autores não coincidentes com a lista de investigadores mais prolixos no campo do ES indicados por Kuzhabekova et al. (2015). 


\section{Considerações Finais}

Apesar da inegável e crescente preocupação das instituições de ES com a formação pedagógica dos seus docentes - como ficou patente no enquadramento onde se enunciam as iniciativas que vêm sendo implementadas ora por unidades orgânicas, ora integradas em políticas ao nível da universidade, ou ainda inserindo-se em políticas de cariz nacional (casos do Reino Unido e da Austrália, por exemplo) - não parece existir interesse homogéneo na comunidade científica no que se refere à construção e/ou circulação do conhecimento acerca da pedagogia do ensino superior. Com efeito, os resultados do estudo de natureza bibliométrica indiciam que a publicação nesta temática não tem, nos anos mais recentes, mantido o fulgor inicial, denotando-se um decréscimo na produção científica a partir de 2014. Estes resultados não deixam de ser surpreendentes quando autores nacionais e internacionais de reconhecido mérito elevam as suas vozes invocando a necessidade de uma formação específica e especializada para os académicos (e.g., Cruz Tomé, 2003; Cunha, 2010; Davidson, 2004; De Ketele, 2003; Elton, 2009; Esteves, 2010; Leite \& Ramos, 2010; Tardif, 2000; Zabalza, 2004, 2007). A este reconhecimento da comunidade científica acrescem as iniciativas de cariz estatal ou institucional, no sentido de implementar políticas e sistemas de formação dos docentes, em alguns casos já com uma longa tradição, de que são exemplo os casos do Reino Unido, Austrália e EUA, países que figuram também nos resultados deste estudo como os mais profícuos.

Ressalve-se que esta análise se limitou à língua inglesa, particularmente às revistas científicas elencadas, na tentativa de a cotejar com a observação das tendências de investigação antes realizada e, por esse motivo, estão ausentes autores portugueses e brasileiros (bem como latino-americanos em geral) que tenham optado por escrever na língua nativa. Não obstante, e face ao crescente interesse em investir na formação pedagógica dos docentes em outros países, que não os que tradicionalmente já aí apostam há décadas, seria expectável que a pesquisa efetuada revelasse uma tendência exponencial de crescimento, envolvendo mais autores e de diferentes nacionalidades. Interpretamos esse abrandamento em razão de uma experiência formativa já devidamente assimilada e tornada usual nas universidades de referência.

Em nosso entender, em países como Portugal, em que há uma fraquíssima aposta na formação pedagógica para o exercício profissional docente no ES, torna-se evidente a necessidade de fazer acompanhar essa mudança estrutural por uma investigação sólida e fortemente ancorada na experiência internacional na área. Só um investimento robusto na produção de conhecimento de âmbito comparado poderá levar as autoridades nacionais e universitárias a consagrar esta formação como necessária e tendencialmente obrigatória. Os investigadores educacionais portugueses têm aqui uma área em que conhecimento e ação se poderão articular com muita intensidade nos próximos anos. 


\section{Referências Bibliográficas}

Altbach, P. G. (2009). Higher education: An emerging field of research and policy. In R. M. Bassett, A. Maldonado-Maldonado (Eds.). International organizations and higher education policy: Thinking globally, acting locally? (pp. 9-25). London: Routledge.

Centra, J. A. (1976). Faculty Development Practices in US Colleges and Universities. Princeton, New Jersey: Educational Testing Service.

Cunha, M. I. (2010). Impasses contemporâneos para a pedagogia universitária no Brasil. In C. Leite (Ed.). Sentidos da pedagogia no ensino superior (pp.63-74). Porto: CllE.

Cruz Tomé, M. A. (2003). Necesidade y objectivos de la formación pedagógica del professor universitário. Revista de Educación, 331, 35-66.

Davidson (2004). Bones of contention: using self and story in the quest to professionalize higher education teaching: an interdisciplinary approach. Teaching in Higher Education, 9(3), 299-310.

De Ketele, J. M. (2003). La Formación didáctica y pedagógica de los profesores universitarios: Luces y Sombras. Revista de Educación, 331, 143-169.

Dearing, R. (1997). The Dearing Report: Higher Education in the Learning Society. The National Committee of Enquiry into Higher Education. Retrieved from http://www.educationengland.org.uk/ documents/dearing1997/dearing1997.html.

Elton, L. (2009). Continuing professional development in higher education: The role of the scholarship of teaching and learning. Arts and Humanities in Higher Education, 8(3), 247-258.

Esteves, M. (2010). Sentidos da inovação pedagógica no ensino superior. In C. Leite (Ed.). Sentidos da Pedagogia no Ensino Superior (pp.45-62). Porto: CIIE.

Gibbs, G. \& Coffey, M. (2004). The impact of training of university teachers on their teaching skills, their approach to teaching and the approach to learning of their students. Active Learning in Higher Education, 5(1), 87-100.

ICED (2014). The Preparation of University Teachers Internationally Report. London: ICED Council. Retrieved from http://icedonline.net/iced-members-area/the-preparation-of-university-teachersinternationally/.

Kuzhabekova, A., Hendel, D. D., \& Chapman, D. W. (2015). Mapping global research on international higher education. Research in Higher Education, 56(8), 861-882.

Leite, C. \& Ramos, R. (2010). Questões da formação pedagógica-didáctica na sua relação com a profissionalidade docente universitária. Alguns pontos para debate. In C. Leite (Ed.). Sentidos da pedagogia no ensino superior (pp.29-45). Porto: CIIE.

Lewis, K. G. (2010). Pathways toward improving teaching and learning in higher education: International context and background. New Directions for Teaching and Learning, 122, 13-23. DOI: 10.1002/ tl.394.

Moses, I. (1987). Educational development units: A cross-cultural perspective. Higher Education, 16(4), 449-479. 
Ouellett, M. L. (2010). Overview of faculty development: History and Choices. In K. J. Gillespie, D. L.

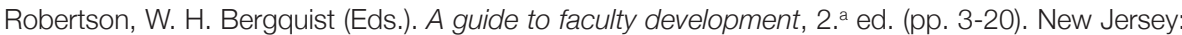
Jossey-Bass.

Sorcinelli, M. D., Austin, A. E., Eddy, P., \& Beach., A. (2006). Creating the Future of Faculty Development: Learning From the Past, Understanding the Present. Bolton, Mass: Anker Press.

Sullivan, L. (1983). Faculty development: A movement on the brink. The College Board Review, 127, 20-21, 29-30.

Tardif, M. (2000). Saberes profissionais dos professores e conhecimentos universitários. Elementos para uma epistemologia da prática profissional dos professores e suas consequências em relação à formação para o magistério. Revista Brasileira de Educação, 13, 5-24.

Zabalza, M. (2004). La enseñaza universitária. El escenario e sus protagonistas (2. a ed.). Madrid: Narcea.

Zabalza, M. (2007). Competências docentes del profesorado Universitario: Calidad y desarrollo profesional. Madrid: Narcea.

\section{Documentos}

VSNU, Association of Universities in the Netherlands (2008). Mutual agreement on a national framework of a University Teaching Qualification System (Overeenkomst Wederzijdse erkenning Basiskwalificatie Onderwijs: BKO). The Hague, Holand.

VSNU, Association of Universities in the Netherlands (2018). Professionalisering van docenten aan de universiteit De BKO en daarna(ast). The Hague, Holand. Retrieved from http://www.vsnu.nl/files/ documenten/Professionalisering\%20van\%20docenten\%20aan\%20de\%20universiteit.PDF.

Commonwealth of Australia (2015). Higher Education Standards Framework (Threshold Standards). Melbourne: Tertiary Education Quality and Standards Agency (TEQSA) Retrieved from: https://www. legislation.gov.au/Details/F2015L01639.

Jorge Ramos do Ó

UIDEF, Instituto de Educação, Universidade de Lisboa

Email: jorge.o@ie.ulisboa.pt ORCID: 0000-0003-1013-9244

Marta Mateus de Almeida

UIDEF, Instituto de Educação, Universidade de Lisboa

Email: mialmeida@ie.ulisboa.pt ORCID: 0000-0003-3108-4289 
Joana Viana

UIDEF, Instituto de Educação, Universidade de Lisboa

Email: jviana@ie.ulisboa.pt

ORCID: 0000-0001-5939-4401

Tatiana Sanches

UIDEF, Instituto de Educação, Universidade de Lisboa

Email: tsanches@fpie.ulisboa.pt

ORCID: 0000-0002-4902-2628

Ana Luísa Paz

UIDEF, Instituto de Educação, Universidade de Lisboa

Email: apaz@ie.ulisboa.pt ORCID: 0000-0003-4848-8183

Correspondência

Tatiana Sanches

Faculdade de Psicologia | Instituto de Educação - Universidade de Lisboa Alameda da Universidade, 1649-013 Lisboa

Data de submissão: Dezembro 2018

Data de avaliação: Março 2019

Data de publicação: Setembro 2019 